\title{
VITESSE DE DISPERSION POUR UNE CLASSE DE MARTINGALES
}

\author{
Thierry DE LA RUE \\ Laboratoire de Mathématiques Raphaël Salem, UMR 6085 CNRS - Université de Rouen, \\ Site Colbert, F76821 Mont-Saint-Aignan Cedex, France
}

Reçu le 17 octobre 2000, revisé le 5 février 2001

ABSTRACT. - In this work we study the dispersion of a martingale $\left(M_{n}\right)$, i.e. the convergence to 0 as $n \rightarrow \infty$ of the concentration function of $M_{n}$, assuming that the martingale differences are bounded, and their conditional variances bounded from below. @ 2002 Éditions scientifiques et médicales Elsevier SAS

Keywords: Martingale; Concentration function

RÉSUMÉ. - On étudie dans ce travail la dispersion d'une martingale $\left(M_{n}\right)$, au sens de la convergence vers 0 quand $n \rightarrow \infty$ de la fonction de concentration de $M_{n}$, sous des hypothèses de majoration des accroissements et de minoration de leurs variances conditionnelles. (๑) 2002 Éditions scientifiques et médicales Elsevier SAS

Mots Clés: Martingale; Fonction de concentration

\section{Introduction}

On s'intéresse ici au comportement en loi d'une martingale $\left(M_{n}\right)_{n \in \mathbb{N}}$ satisfaisant aux hypothèses suivantes : en écrivant $M_{n}$ comme la somme des accroissements de martingale

$$
M_{n}=X_{1}+\cdots+X_{n} \quad\left(M_{0}=0\right),
$$

et en notant $\mathcal{F}_{n}$ la tribu engendrée par $M_{0}, M_{1}, \ldots, M_{n}$, on suppose d'une part que les accroissements $X_{n}$ sont bornés, i.e.

$$
\exists M>0, \forall n \geqslant 1, \quad\left|X_{n}\right| \leqslant M,
$$

et d'autre part que la variance conditionnelle de $X_{n+1}$ sachant $\mathcal{F}_{n}$ est minorée, i.e.

$$
\exists \beta>0, \forall n \geqslant 0, \quad \mathbb{E}\left[X_{n+1}^{2} \mid \mathcal{F}_{n}\right] \geqslant \beta^{2} \quad \text { (p.s.) }
$$


Notons que, de manière évidente, le fait que $\left(M_{n}\right)$ soit une martingale se traduit par la propriété supplémentaire

$$
\forall n \geqslant 0, \quad \mathbb{E}\left[X_{n+1} \mid \mathscr{F}_{n}\right]=0 .
$$

On se propose dans ce travail d'étudier la dispersion d'une telle martingale, mesurée à l'aide de la fonction de concentration de $M_{n}$. Rappelons que la fonction de concentration d'une variable aléatoire réelle $X$, introduite par Paul Lévy [2], est définie pour $l \geqslant 0$ par

$$
Q(X, l) \stackrel{\text { déf }}{=} \sup _{x \in \mathbb{R}} P(x \leqslant X \leqslant x+l) .
$$

On veut montrer que, sous les hypothèses décrites précédemment, $Q\left(M_{n}, l\right) \longrightarrow_{n \rightarrow \infty} 0$ pour tout $l \geqslant 0$, et estimer la vitesse de convergence. Clairement, il suffit de considérer une seule valeur de $l>0$ fixée; on s'intéresse dorénavant au cas $l=2$, et on note pour tout réel $t I_{t} \stackrel{\text { déf }}{=}[t-1, t+1]$.

Le problème abordé ici peut être formulé à l'aide d'un jeu. Un joueur observe un processus aléatoire $\left(X_{1}, X_{2}, \ldots, X_{n}\right)$, sur lequel il peut agir selon la règle suivante : Pour tout $j \in\{1, \ldots, n\}$, après avoir observé $X_{1}, \ldots, X_{j-1}$ ce joueur peut choisir la loi $\mu_{j}$ suivie par $X_{j}$ en respectant les propriétés (1), (2) et (3), c'est-à-dire dans l'ensemble $\mathcal{P}_{\beta, M}$ des lois de probabilités $\mu$ sur $\mathbb{R}$ qui vérifient

$$
\begin{aligned}
& \mu([-M, M])=1, \\
& \int_{\mathbb{R}} x^{2} \mathrm{~d} \mu \geqslant \beta^{2}, \\
& \int_{\mathbb{R}} x \mathrm{~d} \mu=0 .
\end{aligned}
$$

Le joueur gagne si $X_{1}+\cdots+X_{n} \in I_{t}$, pour un certain réel $t$ qu'il aura fixé au départ. On veut ici montrer que, quelle que soit la stratégie du joueur, la probabilité qu'il gagne tend vers 0 quand $n \rightarrow \infty$.

Énonçons maintenant le résultat principal qui sera démontré dans la suite.

THÉORÈME 1.1. - Soit $\left(M_{n}\right)_{n \geqslant 0}$ une martingale avec $M_{0}=0$, et pour tout $n \geqslant 1$, $X_{n} \stackrel{\text { déf }}{=} M_{n}-M_{n-1}$. On suppose que les accroissements $\left(X_{n}\right)$ satisfont aux propriétés $(1)$ et (2). Alors il existe $K>0$ et $\lambda>0$, ne dépendant que de $\beta$ et $M$, tels que pour tout $n \geqslant 1$ et tout $t \in \mathbb{R}$,

$$
P\left(M_{n} \in I_{t}\right) \leqslant K n^{-\lambda} .
$$

Dans le cas où $\left(X_{n}\right)$ est une suite de variables indépendantes identiquement distribuées, le résultat est bien connu avec l'exposant $\lambda=1 / 2$ (voir par exemple [3] p. 49). Pour une martingale, on peut également déduire un tel énoncé avec un exposant $\lambda<1 / 4$ à partir de théorèmes de type Berry-Esseen (comme celui donné dans [1] p. 84), mais qui nécessitent des hypothèses plus fortes sur les variances conditionnelles. Pour le cas qui nous intéresse ici, les conditions imposées à la martingale $\left(M_{n}\right)$ sont trop faibles pour entraîner une quelconque convergence en loi ; aussi il semble assez difficile 
de traiter ce problème avec les outils habituellement utilisés pour l'obtention de résultats fins sur les lois asymptotiques.

\section{Formulation fonctionnelle du problème}

Les réels strictement positifs $\beta$ et $M$ étant fixés, notons $\mathcal{M}_{\beta, M}$ la classe des martingales $\left(M_{n}\right)_{n \geqslant 0}$ vérifiant $M_{0}=0$, et les propriétés (1) et (2). On introduit la suite de fonctions $\left(q_{n}\right)_{n \geqslant 0}$ définie par

$$
\forall n \geqslant 0, \forall t \in \mathbb{R}, \quad q_{n}(t) \stackrel{\text { déf }}{=} \sup _{\left(M_{n}\right) \in \mathcal{M}_{\beta, M}} P\left(M_{n} \in I_{t}\right) .
$$

Démontrer (7) revient donc à trouver $K>0$ et $\lambda>0$ tels que pour tout réel $t$ et tout $n \geqslant 1, q_{n}(t) \leqslant K n^{-\lambda}$.

\subsection{Une relation de récurrence vérifiée par la suite $\left(q_{n}\right)$}

Soit $\left(X_{n}\right)_{n \geqslant 1}$ un processus réel, et $\left(M_{n}\right)_{n} \geqslant 0$ défini par $M_{0} \stackrel{\text { déf }}{=} 0, M_{n} \stackrel{\text { déf }}{=} X_{1}+\cdots+X_{n}$ pour $n \geqslant 1$. On considère aussi, pour $n \geqslant 0, \tilde{M}_{n} \stackrel{\text { déf }}{=} M_{n+1}-X_{1}$; on a aussi $\tilde{M}_{0}=0$, et pour $n \geqslant 1$ on peut écrire $\tilde{M}_{n}=\tilde{X}_{1}+\cdots+\tilde{X}_{n}$, où $\tilde{X}_{k} \stackrel{\text { déf }}{=} X_{k+1}$.

Dire que $\left(M_{n}\right)_{n \geqslant 0}$ est une martingale dans $\mathcal{M}_{\beta, M}$ revient à dire que pour tout $n \geqslant 1$, la loi conditionnelle de $X_{n}$ sachant $X_{1}, \ldots, X_{n-1}$ est presque sûrement dans $\mathcal{P}_{\beta, M}$, ce qui équivaut aux deux propriétés qui suivent :

1. la loi de $X_{1}$ est dans $\mathcal{P}_{\beta, M}$,

2. pour tout $n \geqslant 1$, la loi conditionnelle de $\tilde{X}_{n}$ sachant $\tilde{X}_{1}, \ldots, \tilde{X}_{n-1}$ et $X_{1}$ est presque sûrement dans $\mathcal{P}_{\beta, M}$.

Or, la seconde condition dit exactement que la loi conditionnelle de $\left(\tilde{M}_{n}\right)_{n \geqslant 0}$ sachant $X_{1}$ est presque sûrement celle d'une martingale dans $\mathcal{M}_{\beta, M}$. Finalement, on voit donc que $\left(M_{n}\right)_{n \geqslant 0}$ est une martingale dans $\mathcal{M}_{\beta, M}$ si et seulement si

- la loi $\mu_{1}$ de $X_{1}$ appartient à $\mathcal{P}_{\beta, M}$,

- pour $\mu_{1}$-presque tout $x_{1} \in \mathbb{R}$, la loi du processus $\left(M_{n+1}-x_{1}\right)_{n \geqslant 0}$ sachant $X_{1}=x_{1}$ est celle d'une martingale dans $\mathcal{M}_{\beta, M}$.

On en déduit que pour tout $n \geqslant 0$ et tout $t \in \mathbb{R}$,

$$
q_{n+1}(t)=\sup _{\mu_{1} \in \mathcal{P}_{\beta, M}} \int_{\mathbb{R}} \sup _{\left(M_{n}\right) \in \mathcal{M}_{\beta, M}} P\left(M_{n} \in I_{t-x_{1}}\right) \mathrm{d} \mu_{1}\left(x_{1}\right),
$$

soit, puisque une variable aléatoire $X$ suit une loi dans $\mathcal{P}_{\beta, M}$ si et seulement si la loi de $(-X)$ est dans $\mathcal{P}_{\beta, M}$,

$$
q_{n+1}(t)=\sup _{\mu \in \mathcal{P}_{\beta, M}} \int_{\mathbb{R}} q_{n}(t+x) \mathrm{d} \mu(x) .
$$


Ainsi, la suite $\left(q_{n}\right)$ est entièrement déterminée par la donnée de $q_{0}$, qui n'est autre que $\mathbb{1}_{[-1,1]}$, et la relation de récurrence $q_{n+1}=\tau q_{n}$, où pour $f: \mathbb{R} \rightarrow \mathbb{R}_{+}$mesurable, on pose

$$
\tau f(t) \stackrel{\text { déf }}{=} \sup _{\mu \in \mathcal{P}_{\beta, M}} \int_{\mathbb{R}} f(t+x) \mathrm{d} \mu(x) .
$$

\subsection{Remarques élémentaires sur l'opérateur $\tau$}

Il est immédiat que l'opérateur $\tau$ est croissant : si $f \leqslant g$, alors $\tau f \leqslant \tau g$. On voit aussi facilement que si $f$ est une fonction paire, $\tau f$ est encore une fonction paire, et si $\theta$ est un réel positif, $\tau(\theta f)=\theta \tau f$.

On vérifie également que si $f$ est concave (respectivement convexe) sur l'intervalle $[t-M, t+M]$, alors $\tau f(t) \leqslant f(t)$ (respectivement $\tau f(t) \geqslant f(t)$ ). En fait, si $f$ est de classe $C^{2}$ sur l'intervalle $[t-M, t+M]$, l'inégalité

$$
f(t+x) \leqslant f(t)+x f^{\prime}(t)+\frac{x^{2}}{2} \sup _{[t-M, t+M]} f^{\prime \prime} \quad(x \in[-M, M])
$$

donne, pour $\mu \in \mathcal{P}_{\beta, M}$

$$
\int f(t+x) \mathrm{d} \mu(x) \leqslant f(t)+\frac{1}{2} \sup _{[t-M, t+M]} f^{\prime \prime} \int x^{2} \mathrm{~d} \mu(x) .
$$

On a donc toujours

$$
\tau f(t) \leqslant f(t)+\frac{M^{2}}{2}\left|\sup _{[t-M, t+M]} f^{\prime \prime}\right| .
$$

De plus, si $f$ est concave sur $[t-M, t+M]$ avec $f^{\prime \prime} \leqslant-a(a>0)$, on obtient

$$
\tau f(t) \leqslant f(t)-\frac{a \beta^{2}}{2} .
$$

\section{Action de $\tau$ sur une famille de fonctions}

On considère la fonction $t \longmapsto p(t)$ définie sur $\mathbb{R}$ par

$$
\begin{aligned}
& p(t) \stackrel{\text { déf }}{=} \mathrm{e}^{-|t|} \quad \text { pour }|t| \geqslant 1, \\
& p(t) \stackrel{\text { déf }}{=} b-a t^{2} \quad \text { pour }|t|<1,
\end{aligned}
$$

où $a$ et $b$ sont fixés de telle façon que $p$ soit de classe $C^{1}$ sur $\mathbb{R}$. (Un calcul rapide donne $a=\frac{\mathrm{e}^{-1}}{2}$, et $b=\frac{3}{2} \mathrm{e}^{-1}$.) Puis, pour $\sigma>0$, posons

$$
f_{\sigma}(t) \stackrel{\text { déf }}{=} p(t / \sigma) .
$$

Dans la suite, on cherche à majorer $\tau f_{\sigma}(t) \operatorname{par} \theta f_{\sigma^{\prime}}(t)$, où $\theta$ et $\sigma^{\prime}$ dépendent de $\sigma$. Fixons pour le moment $\sigma$ assez grand (à préciser ultérieurement), et notons pour simplifier $f$ 
au lieu de $f_{\sigma}$. Comme $f$ est paire, $\tau f$ l'est également, et il suffit donc d'étudier $\tau f(t)$ pour $t \geqslant 0$.

\section{1. Étude de $\tau f(t)$ pour $0 \leqslant t \leqslant \sigma-M$}

Sur l'intervalle $[t-M, t+M], f$ coïncide avec un polynôme de degré 2 , de dérivée seconde égale à $-\frac{2 a}{\sigma^{2}}$. L'inégalité (10) donne

$$
\tau f(t) \leqslant f(t)-\frac{a \beta^{2}}{\sigma^{2}} .
$$

Et comme $f(t) \leqslant b=3 a$, on obtient

$$
\tau f(t) \leqslant \theta f(t), \quad \text { avec } \theta \stackrel{\text { déf }}{=} 1-\frac{\beta^{2}}{3 \sigma^{2}} .
$$

\section{2. Étude de $\tau f(t)$ pour $t>\sigma-M$}

Comme $f$ est convexe au-delà de $\sigma$, on ne peut pas espérer majorer $\tau f(t)$ par $\theta f(t)$. Mais $f$ étant décroissante sur $\mathbb{R}_{+}$, on peut envisager une inégalité du type $\tau f(t) \leqslant \theta f((1-\varepsilon) t)$ avec $\varepsilon>0$. On cherche $\varepsilon$ de la forme $\alpha / \sigma^{2}$, avec $\alpha>0$ ne dépendant pas de $\sigma$.

Posons $\varphi(u) \stackrel{\text { déf }}{=} \mathrm{e}^{-u / \sigma}$. On a toujours $f \leqslant \varphi$, donc $\tau f(t) \leqslant \tau \varphi(t)$. Et en remarquant que, pour $\sigma \geqslant M$,

$$
\sup _{[t-M, t+M]} \varphi^{\prime \prime}=\frac{\mathrm{e}^{M / \sigma}}{\sigma^{2}} \varphi(t) \leqslant \frac{\mathrm{e}}{\sigma^{2}} \varphi(t),
$$

on obtient par (9)

$$
\tau f(t) \leqslant \varphi(t)\left(1+\frac{M^{2} \mathrm{e}}{2 \sigma^{2}}\right)
$$

Comparaison de $\varphi(t)$ et $f(t)$

Lorsque $t \geqslant \sigma$, on a $\varphi(t)=f(t)$. Il reste à étudier le cas où $\sigma-M<t<\sigma$. La fonction $f$ est alors un polynôme de degré 2 sur l'intervalle $[t, \sigma]$, avec $f^{\prime \prime}=-\frac{2 a}{\sigma^{2}}$, et on a donc

$$
f(t)=f(\sigma)+(t-\sigma) f^{\prime}(\sigma)-\frac{a}{\sigma^{2}}(t-\sigma)^{2} .
$$

On peut aussi écrire

$$
\varphi(t)=\varphi(\sigma)+(t-\sigma) \varphi^{\prime}(\sigma)+\frac{(t-\sigma)^{2}}{2} \varphi^{\prime \prime}(c),
$$

où $c \in[t, \sigma]$. Or, $f(\sigma)=\varphi(\sigma)$, et $f^{\prime}(\sigma)=\varphi^{\prime}(\sigma)$. En utilisant aussi $\varphi^{\prime \prime}(c)=\frac{\varphi(c)}{\sigma^{2}} \leqslant \frac{\varphi(t)}{\sigma^{2}}$, on obtient

$$
\varphi(t)-f(t)=(t-\sigma)^{2}\left(\frac{1}{2} \varphi^{\prime \prime}(c)+\frac{a}{\sigma^{2}}\right) \leqslant \frac{M^{2}}{2 \sigma^{2}} \varphi(t)+\frac{a M^{2}}{\sigma^{2}},
$$


d'où

$$
\varphi(t)\left(1-\frac{M^{2}}{2 \sigma^{2}}\right) \leqslant f(t)+\frac{a M^{2}}{\sigma^{2}} .
$$

Enfin, comme $f(t) \geqslant f(\sigma)=2 a$, on a

$$
\varphi(t) \leqslant f(t) \frac{1+\frac{M^{2}}{2 \sigma^{2}}}{1-\frac{M^{2}}{2 \sigma^{2}}} .
$$

\section{Minoration de $f\left(t\left(1-\alpha / \sigma^{2}\right)\right)$}

Rappelons que $\alpha$ est un réel strictement positif à préciser ultérieurement, qui ne dépend pas de $\sigma$. On peut donc supposer $\sigma$ assez grand pour que $(\sigma-M)\left(1-\alpha / \sigma^{2}\right)>$ $\sigma / 2$, et $(\sigma+M)\left(1-\alpha / \sigma^{2}\right)>\sigma$. On sait aussi qu'il existe $u \in\left[t\left(1-\alpha / \sigma^{2}\right), t\right]$ tel que

$$
f\left(t\left(1-\alpha / \sigma^{2}\right)\right)=f(t)-\frac{\alpha t}{\sigma^{2}} f^{\prime}(u) \geqslant f(t)-\frac{\alpha}{2 \sigma} f^{\prime}(u) .
$$

Cherchons à estimer plus précisément $f^{\prime}(u)$, en commençant par le cas où $\sigma-M<t<$ $\sigma+M$. Dans ce cas, ou bien $\sigma<u<\sigma+M$, et alors

$$
f^{\prime}(u) \leqslant f^{\prime}(\sigma+M)=-\frac{1}{\sigma} \mathrm{e}^{-(\sigma+M) / \sigma} \leqslant-\frac{1}{\sigma} \mathrm{e}^{-2},
$$

ou bien $\sigma / 2 \leqslant u \leqslant \sigma$, et alors

$$
f^{\prime}(u) \leqslant f^{\prime}(\sigma / 2)=-\frac{\mathrm{e}^{-1}}{2 \sigma} \leqslant-\frac{1}{\sigma} \mathrm{e}^{-2} .
$$

Par ailleurs, $f(t) \leqslant b=\frac{3}{2} \mathrm{e}^{-1}$, et donc

$$
f^{\prime}(u) \leqslant-\frac{2 \mathrm{e}^{-1}}{3 \sigma} f(t) .
$$

Dans le cas plus simple où $t \geqslant \sigma+M$, on a $u>\sigma$, d'où $f^{\prime}(u) \leqslant f^{\prime}(t)=-\frac{1}{\sigma} f(t)$. L'inégalité (15) est donc a fortiori toujours vérifiée.

En reprenant (14), on obtient finalement

$$
f\left(t\left(1-\alpha / \sigma^{2}\right)\right) \geqslant f(t)\left(1+\frac{\alpha \mathrm{e}^{-1}}{3 \sigma^{2}}\right) .
$$

\section{Majoration de $\tau f(t)$}

Les inégalités (12), (13) et (16) donnent

$$
\tau f(t) \leqslant \frac{\left(1+\frac{M^{2} \mathrm{e}}{2 \sigma^{2}}\right)\left(1+\frac{M^{2}}{2 \sigma^{2}}\right)}{\left(1-\frac{M^{2}}{2 \sigma^{2}}\right)\left(1+\frac{\alpha \mathrm{e}^{-1}}{3 \sigma^{2}}\right)} f\left(t\left(1-\alpha / \sigma^{2}\right)\right) .
$$


Choisissons $\alpha$ assez grand pour que

$$
\frac{\alpha \mathrm{e}^{-1}}{3} \geqslant 4 M^{2}+\frac{\beta^{2}}{3} .
$$

Alors, pour $\sigma$ assez grand, on a

$$
\frac{\left(1+\frac{M^{2} \mathrm{e}}{2 \sigma^{2}}\right)\left(1+\frac{M^{2}}{2 \sigma^{2}}\right)}{\left(1-\frac{M^{2}}{2 \sigma^{2}}\right)\left(1+\frac{\alpha \mathrm{e}^{-1}}{3 \sigma^{2}}\right)} \leqslant 1+\frac{4 M^{2}}{\sigma^{2}}-\frac{\alpha \mathrm{e}^{-1}}{3 \sigma^{2}} \leqslant \theta .
$$

Ceci donne finalement, pour $t>\sigma-M$,

$$
\tau f(t) \leqslant \theta f\left(t\left(1-\alpha / \sigma^{2}\right)\right)
$$

Notons enfin que cette inégalité, moins forte que (11), est a fortiori toujours vérifiée pour $0 \leqslant t \leqslant \sigma-M$.

\subsection{Conclusion des calculs précédents}

On a trouvé $\alpha>0$ tel que, si $\sigma$ est assez grand,

$$
\tau f_{\sigma} \leqslant\left(1-\frac{\beta^{2}}{3 \sigma^{2}}\right) f_{\sigma^{\prime}}
$$

où

$$
\sigma^{\prime} \stackrel{\text { déf }}{=} \frac{\sigma}{1-\alpha / \sigma^{2}}
$$

Choisissons maintenant $\sigma_{0}$ assez grand pour vérifier l'inégalité qui précède, et aussi pour que $\alpha / \sigma_{0}<1 / 2$, puis $K_{0}$ assez grand pour que

$$
K_{0} f_{\sigma_{0}} \geqslant q_{0}=\mathbb{1}_{[-1,1]} .
$$

Une récurrence immédiate donne alors, pour tout $n \geqslant 0$,

$$
q_{n} \leqslant K_{n} f_{\sigma_{n}}
$$

où les suites $\left(K_{n}\right)_{n \geqslant 0}$ et $\left(\sigma_{n}\right)_{n \geqslant 0}$ sont définies récursivement par les relations

$$
K_{n+1} \stackrel{\text { déf }}{=}\left(1-\frac{\beta^{2}}{3 \sigma_{n}^{2}}\right) K_{n} \quad \text { et } \quad \sigma_{n+1} \stackrel{\text { déf }}{=} \frac{\sigma_{n}}{1-\alpha / \sigma_{n}^{2}} .
$$

En particulier,

$$
\sup _{t \in \mathbb{R}} q_{n}(t) \leqslant K_{n} b
$$




\section{Étude des suites $\left(K_{n}\right)$ et $\left(\sigma_{n}\right)$}

En utilisant $(1-u)^{-1} \leqslant 1+2 u$ pour $0 \leqslant u \leqslant 1 / 2$, on obtient pour tout $n \geqslant 0$,

$$
\sigma_{n+1} \leqslant \sigma_{n}+\frac{2 \alpha}{\sigma_{n}} .
$$

On en déduit facilement que pour tout $n \geqslant 0, \sigma_{n} \leqslant s_{n}$ où $\left(s_{n}\right)_{n \geqslant 0}$ est définie par $s_{0} \stackrel{\text { déf }}{=} \sigma_{0}$, et $s_{n+1}=s_{n}+2 \alpha / s_{n}$. En remarquant que

$$
s_{n+1}^{2}=s_{n}^{2}+4 \alpha+\frac{4 \alpha^{2}}{s_{n}^{2}} \leqslant s_{n}^{2}+r
$$

où $r$ est une constante réelle, on en déduit l'existence de $\gamma>0$ tel que pour tout $n \geqslant 0$,

$$
\sigma_{n} \leqslant \sqrt{\sigma_{0}^{2}+n r} \leqslant \gamma \sqrt{n+1}
$$

On a donc pour tout $n \geqslant 0$

$$
K_{n+1} \leqslant\left(1-\frac{\beta^{2}}{3 \gamma^{2}(n+1)}\right) K_{n}
$$

d'où

$$
\begin{aligned}
\ln K_{n} & \leqslant \ln K_{0}+\sum_{j=1}^{n} \ln \left(1-\frac{\beta^{2}}{3 \gamma^{2} j}\right) \leqslant \ln K_{0}-\frac{\beta^{2}}{3 \gamma^{2}} \sum_{j=1}^{n} \frac{1}{j} \\
& \leqslant \ln K_{0}-\frac{\beta^{2}}{3 \gamma^{2}} \ln n=\ln \left(K_{0} n^{-\beta^{2} / 3 \gamma^{2}}\right) .
\end{aligned}
$$

Grâce à (18), ceci achève la preuve du théorème 1.1 avec $K \stackrel{\text { déf }}{=} K_{0} b$, et $\lambda \stackrel{\text { déf }}{=} \beta^{2} / 3 \gamma^{2}$.

Ici se pose naturellement la question suivante : à quelle vitesse $\sup _{t} q_{n}(t)$ tend-il réellement vers 0 ? L'exposant $\lambda$ trouvé ci-dessus n'a pas de raison d'être le meilleur possible; notons en particulier que la fonction $p$ utilisée ici a été choisie presque arbitrairement (essentiellement parce qu'avec celle-ci, les calculs s'effectuent assez simplement). Il est possible qu'un raisonnement similaire avec un meilleur choix de $p$ donne un $\lambda$ plus grand.

\section{Affaiblissement des hypothèses}

Peut-on conserver un résultat de dispersion analogue au théorème 1.1 en affaiblissant les hypothèses faites sur la martingale $\left(M_{n}\right)$ ?

Commençons par une remarque facile : la condition $M_{0}=0$ est en fait superflue pour obtenir la conclusion du théorème 1.1. En effet, si $\left(M_{n}\right)$ est une martingale dont les accroissement vérifient (1) et (2), et si on note $\mu_{0}$ la loi de $M_{0}$, il est clair que pour $\mu_{0}$-presque tout $x_{0} \in \mathbb{R}$, la loi du processus $\tilde{M}_{n} \stackrel{\text { déf }}{=} M_{n}-M_{0}$ sachant $M_{0}=x_{0}$ est celle d'une martingale dans $\mathcal{M}_{\beta, M}$. On a alors pour tout réel $t$ 


$$
P\left(M_{n} \in I_{t}\right)=\int_{\mathbb{R}} P\left(\tilde{M}_{n} \in I_{t-x_{0}} \mid M_{0}=x_{0}\right) \mathrm{d} \mu_{0}\left(x_{0}\right) \leqslant \int_{\mathbb{R}} q_{n}\left(t-x_{0}\right) \mathrm{d} \mu_{0}\left(x_{0}\right) \leqslant K n^{-\lambda} .
$$

Étudions maintenant l'affaiblissement des hypothèses (1) et (2).

\subsection{Variances conditionnelles non uniformément minorées}

On se place ici dans le cas où l'hypothèse (1) de majoration des accroissements est toujours vérifiée, mais où l'hypothèse (2) de minoration uniforme des variances conditionnelles n'est plus a priori supposée. On se donne simplement une suite $\left(\beta_{n}\right)_{n \geqslant 1}$ de réels positifs (éventuellement nuls !) tels que

$$
\forall n \geqslant 0, \quad \mathbb{E}\left[X_{n+1}^{2} \mid \mathcal{F}_{n}\right] \geqslant \beta_{n+1}^{2} \quad \text { (p.s.) }
$$

Notons maintenant $\mathcal{M}_{\left(\beta_{n}\right), M}$ la classe des martingales $\left(M_{n}\right)_{n \geqslant 0}$ avec $M_{0}=0$, et vérifiant (1) et (20), et posons

$$
\forall n \geqslant 0, \forall t \in \mathbb{R}, \quad q_{\beta_{1}, \ldots, \beta_{n}}(t) \stackrel{\text { déf }}{=} \sup _{\left(M_{n}\right) \in \mathcal{M}_{\left(\beta_{n}\right), M}} P\left(M_{n} \in I_{t}\right)
$$

on obtient par un raisonnement identique à celui effectué en 2.1

$$
\forall n \geqslant 0, \quad q_{\beta_{1}, \ldots, \beta_{n}}=\tau_{\beta_{1}}\left(\tau_{\beta_{2}} \ldots\left(\tau_{\beta_{n}} \mathbb{1}_{[-1,1]}\right)\right) .
$$

(On utilise ici la notation $\tau_{\beta_{j}}$ au lieu de $\tau$ pour faire apparaître la dépendance par rapport à $\beta_{j}$ : le sup définissant $\tau_{\beta_{j}}$ étant pris lorsque la probabilité $\mu$ décrit la clase $\mathcal{P}_{\beta_{j}, M}$. )

Remarquons que dans les calculs concernant l'action de l'opérateur $\tau$ sur les fonctions $f_{\sigma}$, on peut choisir un réel $\alpha$ vérifiant (17) indépendamment de $\beta$. En effet, pour que $\mathcal{P}_{\beta, M}$ soit non vide on doit avoir $0 \leqslant \beta^{2} \leqslant M^{2}$, et il suffit donc de prendre $\alpha \geqslant 15$ e $M^{2}$. Par ailleurs, on vérifie facilement que le choix de $\sigma$ assez grand pour que la conclusion exposée en 3.3 soit valide ne dépend pas non plus de $\beta \in[0, M]$.

$K_{0}$ et la suite $\left(\sigma_{n}\right)_{n \geqslant 0}$ étant définis comme en 3.3, on a donc de la même façon

$$
\begin{aligned}
q_{\beta_{1}, \ldots, \beta_{n}} & \leqslant K_{0} \tau_{\beta_{1}}\left(\tau_{\beta_{2}} \ldots\left(\tau_{\beta_{n}} f_{\sigma_{0}}\right)\right) \\
& \leqslant K_{0}\left(1-\beta_{n}^{2} / 3 \sigma_{0}^{2}\right) \tau_{\beta_{1}}\left(\tau_{\beta_{2}} \ldots\left(\tau_{\beta_{n-1}} f_{\sigma_{1}}\right)\right) \\
& \leqslant \cdots \leqslant K_{0} b \prod_{j=1}^{n}\left(1-\beta_{j}^{2} / 3 \sigma_{n-j}^{2}\right) .
\end{aligned}
$$

En utilisant toujours l'estimation (19) sur la suite $\left(\sigma_{n}\right)$, on en déduit le théorème plus général qui suit.

THÉORÈME 4.1. - Soit $\left(M_{n}\right)_{n \geqslant 0}$ une martingale avec, pour tout $n \geqslant 1, X_{n} \stackrel{\text { déf }}{=} M_{n}-$ $M_{n-1}$. On suppose que les accroissements $\left(X_{n}\right)$ satisfont aux propriétés $(1)$ et $(20)$. Alors il existe $K>0$ et $\rho>0$ ne dépendant que de $M$, tels que pour tout $n \geqslant 1$ et tout $t \in \mathbb{R}$,

$$
P\left(M_{n} \in I_{t}\right) \leqslant K \exp \left(-\rho\left(\frac{\beta_{1}^{2}}{n}+\frac{\beta_{2}^{2}}{n-1}+\cdots+\frac{\beta_{n-1}^{2}}{2}+\beta_{n}^{2}\right)\right) .
$$


Question. - Le théorème 4.1 affirme que si la suite $\left(\beta_{n}\right)$ de réels positifs est telle que

$$
\frac{\beta_{1}^{2}}{n}+\frac{\beta_{2}^{2}}{n-1}+\cdots+\frac{\beta_{n-1}^{2}}{2}+\beta_{n}^{2} \underset{n \rightarrow \infty}{\longrightarrow}+\infty
$$

alors toute martingale $\left(M_{n}\right)$ dans la classe $\mathcal{M}_{\left(\beta_{n}\right), M}$ vérifie $Q\left(M_{n}, 2\right) \longrightarrow_{n \rightarrow \infty} 0$. Que peut-on dire de la réciproque ? (Autrement dit, la condition (21) est-elle un critère pour avoir cette propriété de dispersion dans $\mathcal{M}_{\left(\beta_{n}\right), M}$ ?)

\subsection{Accroissements non majorés}

Il est facile de voir que la condition (1) de majoration des accroissements ne peut pas être purement et simplement supprimée si on souhaite conserver un résultat de dispersion comparable à celui présenté précédemment. En effet, considérons une suite $\left(Y_{n}\right)_{n \geqslant 1}$ de variables aléatoires indépendantes identiquement distribuées, prenant les valeurs 1 et -1 avec probabilité $1 / 2$. Pour un entier $n_{0} \geqslant 2$ fixé, posons $X_{n} \stackrel{\text { déf }}{=} Y_{n}$ si $n \neq n_{0}$, et $X_{n_{0}} \stackrel{\text { déf }}{=} Y_{n_{0}}\left(Y_{1}+\cdots+Y_{n_{0}-1}+\frac{1}{2}\right)$. Il est immédiat que $\left(M_{n}\right) \stackrel{\text { déf }}{=}\left(X_{1}+\cdots+X_{n}\right)$ est une martingale pour laquelle (2) est vérifié (avec $\beta=\frac{1}{2}$ ), mais $P\left(M_{n_{0}} \in I_{0}\right) \geqslant \frac{1}{2}$, alors que $n_{0}$ peut être arbitrairement grand.

Cependant, on peut imaginer pouvoir remplacer (1) par une condition moins forte, par exemple une majoration de la variance conditionnelle des accroissements. Posons ainsi la question suivante : si $\left(M_{n}\right)$ est une martingale dont les accroissements $\left(X_{n}\right)$ vérifient la condition (2), ainsi que

$$
\exists M>0, \forall n \geqslant 0, \quad \mathbb{E}\left[X_{n+1}^{2} \mid \mathcal{F}_{n}\right] \leqslant M \quad \text { (p.s.), }
$$

a-t-on alors nécessairement $\sup _{t \in \mathbb{R}} P\left(M_{n} \in I_{t}\right) \longrightarrow_{n \rightarrow \infty} 0$ ?

\section{RÉFÉRENCES}

[1] Hall P., Heyde C.C., Martingale Limit Theory and its Application, Academic Press, 1980.

[2] Lévy P., Théorie de l'Addition des Variables Aléatoires, Gauthier-Villars, Paris, 1954.

[3] Petrov V.V., Sums of Independent Random Variables, Springer-Verlag, Berlin, 1975. 